\title{
Induced Quantized Spin Current in Vacuum
}

\author{
Chong-Sun Chu $\odot$ and Chun-Hei Leung \\ Department of Physics, National Tsing-Hua University, Hsinchu 30013, Taiwan \\ and Center for Theory and Computation, National Tsing-Hua University, Hsinchu 30013, Taiwan
}

(Received 17 May 2021; revised 25 June 2021; accepted 3 August 2021; published 7 September 2021)

We uncover a fundamental effect of the QED vacuum in an external electromagnetic (EM) field. We show that the quantized vacuum of electrons is spin polarized by the EM field and manifests as a vacuum spin current. An experiment is proposed to measure the spin torque exerted by the spin current by measuring the twisted angle of the director axis of a nematic liquid crystal.

DOI: 10.1103/PhysRevLett.127.111601

Introduction.-The quantum nature of vacuum is a fascinating place to look for novel physical phenomena. For example, the large scale fluctuation of the Universe is supposed to emerge from the quantum ripples of the near de Sitter vacuum in the inflationary universe [1]. In high energy physics, the metastability of the Higgs vacuum [2] has nontrivial cosmological consequences and could provide a new observational window to particle physics well beyond what collider experiments can achieve.

Recently, it was found that the vacuum fluctuations of boundary QED system in the presence of an external magnetic field result in a magnetization current near its boundary $[3,4]$. This nontrivial electromagnetic response of the vacuum is due to the electric charges carried by the virtual electrons and positrons of the theory. As these quantum fluctuations also carry spin, it is natural to ask if and how the quantum fluctuation of the spin degrees of freedom of the vacuum would manifest in observation.

In this Letter, we show that due to the renormalization effect of the spin-orbit coupling of the electrons, the quantized vacuum can become spin polarized in the presence of an applied EM field and results in a spin current; see Eq. (25). The vector spin current is orthogonal to the applied electric field and the observed spin polarization. We also discuss briefly how it may be observed experimentally using a setup of nematic liquid crystal.

Definition of spin current.-The transportation of the spin of electrons is described by a spin current. Classically, the spin current is given by the 3-tensor $J^{i j}=n v^{i} s^{j}$, where $v^{i}$ is the velocity of the electron, $s^{j}$ is the spin, $n$ is the particle number density, and $i, j=1,2,3$ denotes the spatial directions. Quantum mechanically, the spin

Published by the American Physical Society under the terms of the Creative Commons Attribution 4.0 International license. Further distribution of this work must maintain attribution to the author(s) and the published article's title, journal citation, and DOI. Funded by SCOAP . polarization of electrons is represented by the Pauli matrices $\sigma^{i}$ in the rest frame of the electron and a definition of the spin current:

$$
J^{\mu i}=\frac{1}{2}\left(v^{\mu} \sigma^{i}+\sigma^{i} v^{\mu}\right), \quad J^{0 i}=\sigma^{i},
$$

in terms of a symmetrization of the velocity and the spin has been proposed by Rashba [5]. Although this works well for a nonrelativistic system, the generalization to the relativistic case requires the use of a relativistic spin operator. Such an operator was constructed long ago by Bargmann and Wigner [6] for the free Dirac theory, and it reads

$$
T_{\mathrm{BW}}^{i}=\beta \Sigma^{i}+\gamma^{5} p^{i} / m, \quad T_{\mathrm{BW}}^{0}=\boldsymbol{\Sigma} \cdot \mathbf{p} / m,
$$

where $\beta=\gamma^{0}, \alpha^{i}=\beta \gamma^{i}, \gamma^{5}=i \gamma^{0} \gamma^{1} \gamma^{2} \gamma^{3}$ is the chirality matrix and $\Sigma^{i}=(i / 2) \varepsilon^{i j k} \gamma^{j} \gamma^{k}$. We will adopt the particle physics convention of metric signature $(+,-,-,-)$ in this Letter. The Bergmann-Wigner spin operator $T^{\mu}$ is a Lorentz 4-vector [7], it acts as the generator of the little group of the Poincare group. That this is so is because it is indeed equivalent to the Pauli-Lubanski operator

$$
W^{\mu}=-\frac{1}{2 m} \varepsilon^{\mu \alpha \beta \nu} J_{\alpha \beta} p_{\nu} .
$$

This can be seen by noticing that the spin angular momentum generator is given by $J_{\alpha \beta}=(i / 2)\left[\gamma_{\alpha}, \gamma_{\beta}\right]$ in the spinor representation. It is then easy to see that Eq. (3) is equal to the spin operator Eq. (2) on shell.

In this Letter, we are interested in the renormalization phenomena of the QED vacuum in the presence of an external electromagnetic field $A^{\mu}$. As the velocity is represented by $v^{\mu}=\beta \gamma^{\mu}$ in the Dirac theory, we propose the following definition of spin current in quantum field theory:

$$
J_{S}^{\mu \alpha}(x):=\bar{\psi}(x) S^{\mu \alpha} \psi(x),
$$


where

$$
S^{\mu \alpha}:=\frac{1}{2}\left[\gamma^{\mu} T^{\alpha}(x)+T^{\alpha}(x) \gamma^{\mu}\right]
$$

and $T^{\alpha}$ is the covariant Bergmann-Wigner spin operator in a background electromagnetic field:

$$
T^{i}=\beta \Sigma^{i}+\gamma^{5} \frac{\pi^{i}}{m}, \quad T^{0}=\frac{1}{m} \boldsymbol{\Sigma} \cdot \pi .
$$

Here $\pi^{i}=p^{i}-e A^{i}$ is the covariant momentum and $e>0$ is the magnitude of the electric charge. Similar definitions $[8,9]$ have been considered before. Our definition is better justified since we have adopted a symmetrization prescription such that Eq. (4) reduces to the one [Eq. (1)] of Rashba in the nonrelativistic limit. A further justification of our definition (4) can be obtained by noticing that the current Eq. (4) satisfies the conservation law

$$
\partial_{\mu} J^{\mu \alpha}=\frac{e}{m} \mathcal{S}_{\nu} F^{\nu \alpha},
$$

with a source term. Here $\mathcal{S}^{\nu}$ is the density defined by

$$
\mathcal{S}^{i}:=\psi^{\dagger} \Sigma^{i} \psi, \quad \mathcal{S}^{0}:=\psi^{\dagger} \gamma^{5} \psi
$$

We note in passing that $J^{\mu \alpha}$ is not a Noether current, but Eq. (7) is obtained from the fermion equation of motion. For the spatial directions $\alpha=i$, Eq. (7) gives explicitly

$$
\frac{\partial \rho^{i}}{\partial t}+\nabla \cdot \boldsymbol{J}^{i}=\frac{e}{m}\left((\mathcal{S} \times \boldsymbol{B})^{i}+\mathcal{S}^{0} E^{i}\right) .
$$

Here $\rho^{i}:=J^{0 i}$ and $\boldsymbol{J}^{i}:=\mathbf{e}_{k} J^{k i}$. It is clear that $\rho^{i}$ gives the spin polarization density. As a result, $\boldsymbol{J}^{i}$ does admit the correct interpretation as a current density for spin polarization in the $i$ th direction. We also note that $-\nabla \cdot J^{i}$ and the EM terms on the right-hand side of Eq. (9) can be interpreted as the spin torque from the matters and the external EM fields. Classically in a vacuum, $\mathcal{S}^{\mu}$ vanishes and the spin current respects the continuity equation

$$
\frac{\partial \rho^{i}}{\partial t}+\nabla \cdot \boldsymbol{J}^{i}=0
$$

We will show in this Letter that, in the presence of a background electromagnetic field, the spin current $\boldsymbol{J}^{i}$ and the spin density $\rho^{i}$ becomes nonzero due to the polarization effect of the electromagnetic field on the quantum fluctuations of the vacuum. Nevertheless the conservation law (10) is still satisfied at the quantum mechanical level.

We end this section with a couple of remarks. (i) Properly speaking, the current Eq. (4) measures the flow of spin polarization and should be called a spin polarization current. We will follow the common practice of the literature, e.g., Ref. [5], and refer to it as the spin current. To get the spin momentum current, one needs to multiply Eq. (4) with $\hbar / 2$ of the spin angular momentum of each fermion. (ii) We note that in the nonrelativistic limit, the magnetic dipole coupling and the spin-orbit coupling term in the Dirac Hamiltonian

$$
H_{E, B}=-\frac{e}{2 m} \boldsymbol{\Sigma} \cdot \boldsymbol{B}-\frac{e}{4 m^{2}} \boldsymbol{\Sigma} \cdot \mathbf{E} \times \mathbf{p}
$$

can be written in the form

$$
H_{E, B}=-\frac{e}{8 m} \varepsilon_{\mu \nu \lambda \sigma} J^{\mu \nu} F^{\lambda \sigma},
$$

where $J^{\mu \nu}$ here is the spin current (1) in the nonrelativistic limit. The form (12) shows clearly that the spin current, at least in the nonrelativistic limit, couples to the EM field strengths instead of the EM potentials like the electric current $J^{\mu}=-e \bar{\psi} \gamma^{\mu} \psi$. (iii) Equation (12) also shows that a certain amount of energy is needed to generate the stated vacuum spin current and this is supplied by the external power source that maintains the background EM field configuration. (iv) Finally, we remark that for a quantum field theory in curved space, there is a current $J_{\mu}^{a b}$ that couples to the spin connection $\omega_{\mu}^{a b}$. Here $a, b=0,1,2,3$ refer to the frame indices. This current couples to gravity and is also sometimes referred to as a spin current in the respective community. However we emphasize that this is different from the spin current we introduced in this Letter.

Vacuum expectation of spin current.-In quantum field theory, the spin current (4) is a composite operator which needs to be renormalized. We are interested in the vacuum expectation value (vev) of the spin current in a background electromagnetic field $A_{\mu}$. This can be computed in perturbation theory as

$$
\left\langle J^{\mu \alpha}(x)\right\rangle_{A}=-i e \int d^{4} y\left\langle J^{\mu \alpha}(x) J^{\beta}(y)\right\rangle A_{\beta}(y)+O\left(A^{2}\right),
$$

or, it can be written in the momentum space as

$$
\left\langle J^{\mu \alpha}(q)\right\rangle_{A}=e T^{\mu \alpha \beta}(q) A_{\beta}(q)+O\left(A^{2}\right),
$$

where $T^{\mu \alpha \beta}(q)$ is the Green's function

$$
T^{\mu \alpha \beta}(q):=-i \int d^{4} x e^{i q x}\left\langle J^{\mu \alpha}(x) J^{\beta}(0)\right\rangle .
$$

At 1-loop, $T^{\mu \alpha \beta}(q)$ is given by

$T^{\mu \alpha \beta}(q)=-i \int \frac{d^{4} p}{(2 \pi)^{4}}(-1) \operatorname{tr}\left(S^{\mu \alpha} \frac{i}{\not p-m} \gamma^{\beta} \frac{i}{\not p+\not q-m}\right)$, 
where $S^{\mu \alpha}$ is given by Eq. (5). The trace of the gamma matrices can be simplified and we obtain

$$
T^{\mu 0 \beta}=0, \quad T^{\mu i \beta}=-4 m \varepsilon^{i \mu \delta \beta} q_{\delta} I(q), \quad i=1,2,3,
$$

where $I(q)$ is the momentum function defined by

$$
I(q):=\int \frac{d^{4} p}{(2 \pi)^{4}} \frac{1}{p^{2}-m^{2}} \frac{1}{(p+q)^{2}-m^{2}} .
$$

Note that $I(q)$ and hence the Green's function is logarithmic divergent. This is due to the singular product of local quantum fields in the expression (4) for the spin current operator. In order to give a proper definition of this composite operator, one need to isolate the divergent terms with a regularization scheme and subtract it away with counterterms in the Lagrangian [10]. Regularizing Eq. (18), we obtain

$I(q)=\frac{i}{16 \pi^{2}}\left(\log \frac{\Lambda^{2}}{\mu^{2}}-1+\log \frac{\mu^{2}}{m^{2}}-h\left(\frac{q^{2}}{m^{2}}\right)+\cdots\right)$

for a momentum cutoff regularization, and

$I(q)=\frac{i}{16 \pi^{2}}\left(\frac{2}{\epsilon}-\gamma_{E}+\log \frac{4 \pi \mu^{2}}{m^{2}}-h\left(\frac{q^{2}}{m^{2}}\right)+\cdots\right)$

for dimensional regularization to $d=4-\epsilon$ dimension. Here $\cdots$ denotes terms of order $O\left(1 / \Lambda^{2}\right)$ or $O(\epsilon)$, and $h(x)$ is the function

$$
h(x):=\int_{0}^{1} d \xi \ln [1+\xi(1-\xi) x]
$$

with $h(0)=0$. We can now subtract away the divergence with a counterterm and obtain

$\left\langle J_{R}^{\mu i}(x)\right\rangle_{A}=-\frac{e m c}{8 \pi^{2} \hbar} \varepsilon^{i \mu \delta \beta}\left(\log \frac{m^{2}}{\mu^{2}}+a_{J}+h\left(\frac{\square}{m^{2}}\right)\right) F_{\delta \beta}(x)$

and $\left\langle J_{R}^{\mu 0}(x)\right\rangle_{A}=0$. Here $\mu$ is the standard RG scale of the QFT and $a_{J}$ is an independent arbitrary constant that is due to the arbitrariness in the choice of the finite part of the counterterm for the composite operator $J^{\mu i}$.

To uniquely fix the finite part (i.e., fixing $a_{J}$ ) and hence the definition of the renormalized spin current, a normalization condition is required. Physically, if the electron mass is sent to infinity, then the quantum loop effects are completely suppressed and the renormalized spin current mush vanish. In a theory with a cutoff $\Lambda$, the fermion loop effects are now suppressed as $m$ approaches this scale. As a result, we have the decoupling condition

$$
\lim _{m \rightarrow \Lambda}\left\langle J_{R}^{\mu i}\right\rangle_{A}=O\left(\frac{1}{\Lambda}\right)
$$

This serves as a natural normalization condition for the renormalized spin current. However extra care is needed for QED where there is a Landau pole and the UV cutoff cannot be taken to exceed that. In fact, the positiveness of the beta function $\beta(\alpha)=2 \alpha^{2} / 3 \pi\left(\alpha=e^{2} / 4 \pi \hbar c\right.$ is the fine structure constant) give rises to the RG flow for the coupling

$$
\frac{1}{\alpha(\mu)}-\frac{1}{\alpha\left(\mu_{0}\right)}=-\frac{2}{3 \pi} \log \frac{\mu}{\mu_{0}} .
$$

This implies the presence of a cutoff scale $\Lambda_{L}=m e^{3 \pi / 2 \alpha}$, the Landau scale, where the bare coupling $\alpha\left(\Lambda_{L}\right)$ becomes infinite. The Landau scale represents the highest possible UV cutoff one may utilize in the renormalization program of QED. Taking $\Lambda=\Lambda_{L}$ in Eq. (23), the renormalization constant $a_{J}=-\log \Lambda_{L}^{2} / \mu^{2}$ is fixed up to $O\left(1 / \Lambda_{L}\right)$ terms, which we will ignore. Restoring the units of $c / \hbar^{2}$ [11], we finally obtain

$$
\left\langle J_{R}^{\mu i}(x)\right\rangle_{A}=\frac{e m c}{8 \pi^{2} \hbar^{2}}\left(\frac{3 \pi}{\alpha}-h\left(\frac{\square}{m^{2}}\right)\right) \varepsilon^{i \mu \delta \beta} F_{\delta \beta} .
$$

Note that the current Eq. (25) is conserved due to the Bianchi identity of the EM field. For a classical electromagnetic background in vacuum, it is $\square F_{\alpha \beta}=0$ and we have

$$
\left\langle J_{R}^{\mu i}(x)\right\rangle_{A}=\frac{3 e m c}{8 \pi \hbar^{2} \alpha} \varepsilon^{i \mu \delta \beta} F_{\delta \beta},
$$

or, in terms of components explicitly

$$
\begin{aligned}
\left\langle J_{R}^{j i}\right\rangle_{A} & =-\frac{3 e m c}{4 \pi \hbar^{2} \alpha} \varepsilon^{j i k} E^{k}, \\
\left\langle J_{R}^{0 i}\right\rangle_{A} & =\frac{3 e m c}{4 \pi \hbar^{2} \alpha} B^{i} .
\end{aligned}
$$

The results (25), (26), (27) are the main results of this Letter. For the rest of the Letter, we will be focusing on the case (26) of a classical background. However, for generality we present the result (25) to cover the situation where the background EM fields go beyond the Maxwell description; for example, if quantum nonlinear corrections of QED is included, or if the classical EM fields are coupled to other background fields (e.g., an axion background).

A couple of remarks are in order. (i) Note that our result is independent of the adopted regularization scheme. Apart from the cutoff regularization and the dimensional regularization, one can also do the heat-kernel regularization and obtain the same intermediate result (22), and the final result (25) after imposing the normalization condition. 
(ii) It is interesting that the normalization condition (23) fixes $a_{J}$ in terms of the renormalization scale $\mu$. In the end, $\mu$ remains free in the QFT and it is remarkable that the renormalized spin current is independent of it. (iii) We remark that the renormalization condition (23) is essentially nonperturbative in nature as the resulting prediction (25) scales inversely with $\alpha$ and is not smoothly connected with the free theory result.

It is instructive to compare our effect with the famous Schwinger effect [12,13], which refers to the production of electron-positron pairs under the influence of an applied electric field. The Schwinger effect is nonperturbative and requires a strong electric field stronger than the critical field strength $E \gtrsim E_{\text {critical }}=m^{2} c^{3} /(e \hbar)$ in order to produce an observable amount of particle pairs. Unlike the Schwinger effect, the spin current predicted in this Letter is a nonperturbative consequence of the nontrivial spin polarization of the vacuum. There is no real production of particles involved. Our result is also different from the analysis of Ref. [9] where the Schwinger-effect-produced electric current is acted on by a second electric field to produce a spin current. In this case, the spin current is generated in a similar manner as in an ordinary material sample except that the source electric current has a nonperturbative origin and so very small in magnitude.

In the above we have considered pure QED. In a more realistic setting where QED is embedded as the low energy part of a consistent high energy theory, e.g., a grand unified theory or string theory when gravity is included, the Landau pole would be replaced by the corresponding GUT or Planck scale. In this case, the 1-loop results (25) will have the factor $3 \pi / \alpha$ replaced by $3 \pi\left(1 / \alpha-1 / \alpha_{G}\right)$, where $\alpha_{G}$ is the fine structure coupling at the unification scale. It is interesting that the vacuum expectation value of the spin current actually provides a probe to the UV physics. The value of $1 / \alpha_{G}$ is model dependent. For example, for the MSSM GUT, one has [14] $1 / \alpha_{G} \simeq 24.3$ and $M_{G} \simeq 2 \times 10^{16} \mathrm{GeV}$. In any case, $1 / \alpha_{G}$ is expected to be small compared to the observed $1 / \alpha$ at the electron mass scale. In the following, we will continue to analyze the result (25) for pure QED, but keeping in mind the overall magnitude of the spin current may be different from Eq. (25) by a small fraction.

Physical picture.--It may appear strange that the switching on of an electric field in vacuum can produce an observable spin current (27). However the physical origin of the spin current can be easily understood in turns of the spin-orbit coupling of the quantum fluctuation of the vacuum. To see this, let us consider the nonrelativistic expansion of Dirac's Hamiltonian up to $O\left(p^{4}\right)$ where a spin-orbit coupling term arises, $H_{\mathrm{SO}}=-\left[(e \hbar) /\left(4 m^{2} c^{2}\right)\right] \boldsymbol{\Sigma}$. $\mathbf{E} \times \mathbf{p}$. It is well known that the spin-orbit coupling term allows impurity in a material to scatter the electrons in a spin-dependent way (skew scattering) and generates a spin current $[15,16]$. The spin-orbit coupling also give rises to a side jump [17,18] described by the additional velocity

$$
\mathbf{v}_{\mathrm{SO}}=\frac{\partial H_{\mathrm{SO}}}{\partial \mathbf{p}}=-\lambda \boldsymbol{\Sigma} \times \mathbf{E}, \quad \lambda:=\frac{e \hbar}{4 m^{2} c^{2}}
$$

which produces a spin-dependent shift to the trajectory of the electrons and contributes to the spin current.

In vacuum, there are no charge carriers to start with, but virtual pairs of electrons and positrons can be created and live for a short time before they annihilate back. In standard QFT without background field, such vacuum polarization processes give rise to the running of couplings and scaling of dimensions in the theory. However, new observable effects may result in the presence of a background field. Without an electric field, the created electron-positron pair will move away from each other with opposite velocities $\pm u_{0}^{j}$ due to momentum conservation. When an $E$ field is turned on, the particles acquire an additional velocity (28) in accordance with their spin states. The velocities of the virtual $e^{ \pm}$ particles are given by $u^{j}= \pm u_{0}^{j}+v_{ \pm}^{j}$, where $v_{ \pm}^{j}=$ $-\lambda \varepsilon^{j k l} \sigma_{ \pm}^{k} E^{l}$ and $\sigma_{ \pm}^{i}$ are the spin operators for the $e^{+}$and $e^{-}$respectively. Take a measurement of the spin in the $i$ th direction, the expectation value of the $j$ th component of the spin current $\left\langle J^{j i}\right\rangle=\left\langle n \sigma_{+}^{i}\left(u_{0}^{j}+v_{+}^{j}\right)\right\rangle+\left\langle n \sigma_{-}^{i}\left(-u_{0}^{j}+v_{-}^{j}\right)\right\rangle$ gives

$$
\left\langle J^{j i}\right\rangle=-2 n \lambda \varepsilon^{j i k} E_{k},
$$

where $n$ is the number density of the virtual pairs. In deriving Eq. (29), we have used $\left\langle\sigma_{ \pm}^{i}\right\rangle=0$ and the correlations $\left\langle\sigma_{ \pm}^{i} \sigma_{ \pm}^{j}\right\rangle=\delta^{i j} \mathbf{1}$ for the vacuum. As a result, the free field velocity parts in Eq. (29) make no contribution, while the anomalous velocity part of the virtual $e^{ \pm}$contribute equally to the spin current. With the estimate that there is one virtual pair within each volume of Compton wavelength, $n \sim(m c / \hbar)^{3}$, we recover the result (27a) up to a numerical coefficient (including the sign) of order 10. It is clear from this quantum mechanical argument that the induced spin current is a result of the nontrivial spin-spin correlation imprinted on the vacuum due to the applied electric field. This is similar to the induced current [3,4] and the induced Fermi condensate $[19,20]$ which are due to nontrivial magnetization and condensation of the quantized vacuum as a result of external fields.

Proposed experiment.-The induced spin current may be observed by measuring the torque exerted by the spin current on a probe placed in the vacuum. Consider an infinitesimal volume element $\delta V=\delta x \delta y \delta z$ in the interior of a probe placed under the influence of an external EM field. The spin momentum torque acting on $\delta V$ is $\tau^{i}=-\hbar / 2 \int_{\delta V} d^{3} x \nabla \cdot\left\langle\boldsymbol{J}^{i}\right\rangle$. Using the result (27) for a classical EM field in vacuum, we have 


$$
\tau^{i}=\frac{3 e m c}{8 \pi \hbar \alpha} \dot{B}^{i} \delta V
$$

where $\bar{B}^{i}:=\delta V^{-1} \int_{\delta V} \partial B^{i} / \partial t$ is the average rate of change of the magnetic field over the volume $\delta V$. We will be using the SI units from now on and hence a factor of $c$ appears in Eq. (30). For a $B$ field pointing in the $z$ direction described by a wave of the form

$$
B_{z}=B_{0} g\left(t-\frac{x}{c}\right)
$$

where $g$ is as in Fig. 1, we obtain

$$
\tau^{z}=\frac{3 e m c}{8 \pi \hbar \alpha} B_{0} f \delta V,
$$

where $f:=1 / T_{1}-1 / T_{2}$. For an external field with $B_{0}=100 \mathrm{G}, f=1000 \mathrm{~Hz}$, the quantum spin torque per unit volume is

$$
\tau^{z} / \delta V=6.5 \times 10^{-5} \mathrm{Nm}^{-2} .
$$

Not all of the vacuum torque is transferred to the probe. Physically the angular momentum density $\mathcal{J}_{0}^{i}=(\hbar / 2) \rho^{i}$ acquired by the vacuum generates a vacuum magnetization $\boldsymbol{M}_{0}=(e / m) \mathcal{J}_{0}$ and this corresponds to quantum addition to the $B$ field, $\Delta \boldsymbol{B}=\mu_{0} \boldsymbol{M}_{0}$. For a material probe with magnetic susceptibility $\chi$, the $B$ field generates a probe magnetization $\boldsymbol{M}=\chi \boldsymbol{M}_{0} /(1+\chi)$. These are just the Barnett effect and the Einstein-de Haas effect for the interplay between angular momentum and magnetization [21]. This implies the probe receives a torque $\tau_{p}^{i}$ :

$$
\tau_{p}^{i}=\eta \tau^{i},
$$

where $\eta=4 \pi \chi /(1+\chi) \approx \chi$ as $|\chi| \ll 1$. The spin torque on the probe is thus tiny. Nevertheless, the twisting effect may be observable by using a liquid crystal which is known to be exceptional in sensitivity for torque measurement. In fact, recently the Casimir torque exerted on the surface of a liquid crystal [22] has been observed successfully [23]. We propose here a similar setup to observe the spin torque arising from the spin current.

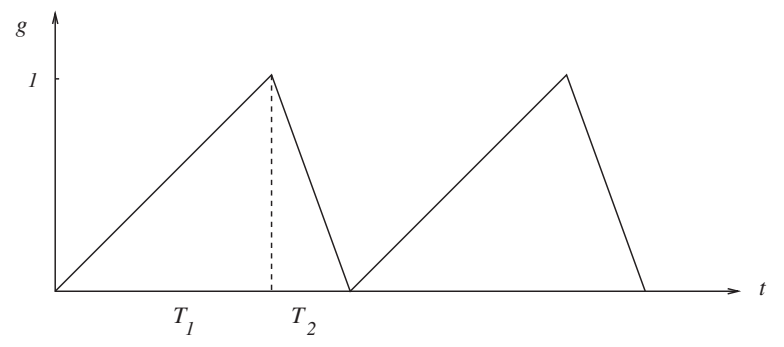

FIG. 1. Waveform of the $B$ field.
Consider a layer of nematic liquid crystal, e.g., 4-cyano40-pentylbiphenyl (5CB), placed in a vacuum with one side $(z=0)$ anchored at a glass interface and the rest of the liquid crystal $\left(0<z \leq L_{z}\right)$ sits in the vacuum cavity, influenced by the external EM field; see Fig. 2. We place the liquid crystal such that its director axis is always in the $x y$ plane, i.e., $\boldsymbol{n}(z)=[\cos \theta(z), \sin \theta(z), 0]$, where $\theta(z)$ describes the orientation of the liquid crystal molecules with respect to the $x$ axis. Because of the action of the spin torque (32), the molecules try to orient themselves correspondingly. An equilibrium configuration is attained when this torque is balanced by the restoring elastic torque of the liquid crystal.

Suppose there is no bend or splay of the liquid crystal, then only the twist contributes to distorting energy density $u_{d}=(k / 2)[(\partial \theta / \partial z)]^{2}$, where $k$ is the twist elastic constant. For example, $k=3.6 \mathrm{pN}$ for the crystal $5 \mathrm{CB}$. The elastic energy stored in $\delta V$ is thus

$$
E_{d}[\theta(z)]=A \int_{z}^{z+\delta z} d z \frac{k}{2}\left(\frac{\partial \theta}{\partial z}\right)^{2},
$$

where $A=\delta x \delta y$ is the area of the element. This give rises to a restoring torque

$$
\tau_{d}(z)=\frac{\delta E_{d}}{\delta \theta(z)}=-k \frac{\partial^{2} \theta}{\partial z^{2}} \delta V
$$

This is in analogy with Newton's second law $F=m \ddot{x}$ for the inertia. The equilibrium is attained when Eq. (34) is balanced out by the restoring torque [24]. Note that the volume factor cancels out. This gives

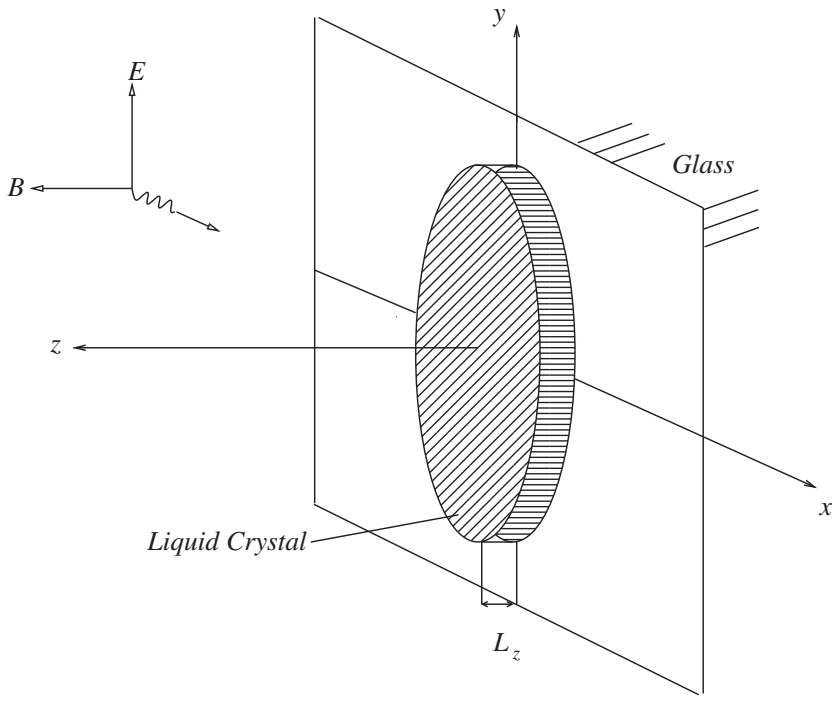

FIG. 2. The director axis of a liquid crystal probe is twisted by the induced spin torque exerted by the vacuum. 


$$
\theta(z)=\frac{\beta}{2} z^{2}, \quad \beta:=\frac{3 \pi \chi}{2 \alpha} \frac{m f_{B} f}{k \lambda_{C}},
$$

where $f_{B}:=e B_{0} /(2 \pi m)$ is the cyclotron frequency and $\lambda_{C}$ is the Compton wavelength. In deriving Eq. (37), we have taken the boundary conditions $\theta=\partial_{z} \theta=0$ at the glass contact $z=0$.

To enhance the detection of the twisted angle, a thicker liquid crystal layer and a stronger magnetic field is preferred. However, both of these are limited by the properties of the liquid crystal. In order for the liquid crystal to be able to register the torque, the response time of the liquid crystal should be smaller than $T_{1}$ and $T_{2}$. This means $f$ is upper bounded by the response frequency $f_{r}$ : $f<f_{r}$. However, the response frequency is limited by the thickness as generally the response time of the liquid crystal increases with the thickness, linearly or quadratically depending on the voltage [25]. As for the EM field, the field strengths cannot be too strong as otherwise the crystal may be driven into a Freederick transition [25]. Nematic liquid crystals are typically diamagnetic with $\chi$ of the order of $10^{-5}$ [25]. Commercial nematic liquid crystal has a $f_{r}$ in the range of $100 \mathrm{~Hz}$ and thickness of the order of $10 \mu \mathrm{m}$. As an estimate, consider a setup with a $100 \mathrm{G} B$ field and a nematic liquid crystal with an elastic constant $k=3.6 \mathrm{pN}$, thickness $L_{z}=1 \mathrm{~mm}$, a response frequency of $1 \mathrm{kHz}$. The total twist angle accumulated over the thickness of the probe is

$$
\Delta \theta=0.005^{\circ} \cdot \frac{\chi}{10^{-5}} \cdot \frac{3.6 \mathrm{pN}}{k} \cdot \frac{B_{0}}{100 \mathrm{G}} \cdot \frac{f}{1 \mathrm{kHz}} \cdot\left(\frac{L_{z}}{1 \mathrm{~mm}}\right)^{2} .
$$

This looks feasible. It will be interesting to perform an experiment to make observation of the spin current predicted in this Letter. The quantum spin current could have a wide range of applications, from novel theoretical properties of physical system, e.g., dark energy, to practical effects on the workings of micro-machined device.

We acknowledge support of this work by the Grant No. 107-2119-M-007-014-MY3 of the Ministry of Science and Technology of Taiwan.

[1] A. R. Liddle and D. H. Lyth, Cosmological Inflation and Large-Scale Structure (Cambridge University Press, Cambridge, England, 2000).

[2] F. Bezrukov, J. Rubio, and M. Shaposhnikov, Living beyond the edge: Higgs inflation and vacuum metastability, Phys. Rev. D 92, 083512 (2015).

[3] C. S. Chu and R. X. Miao, Weyl Anomaly Induced Current in Boundary Quantum Field Theories, Phys. Rev. Lett. 121, 251602 (2018).
[4] C. S. Chu and R. X. Miao, Anomalous transport in holographic boundary conformal field theories, J. High Energy Phys. 07 (2018) 005.

[5] E. I. Rashba, Spin currents in thermodynamic equilibrium: The challenge of discerning transport currents, Phys. Rev. B 68, 241315(R) (2003).

[6] V. Bargmann and E. P. Wigner, Group theoretical discussion of relativistic wave equations, Proc. Natl. Acad. Sci. U.S.A. 34, 211 (1948).

[7] See, for example, D. M. Fradkin and R. H. Good, Jr., Electron polarization operators, Rev. Mod. Phys. 33, 343 (1961) for a review of the construction and properties of the Bergmann-Wigner spin operator.

[8] A. Vernes, B. L. Gyorffy, and P. Weinberger, Spin currents, spin-transfer torque, and spin-Hall effects in relativistic quantum mechanics, Phys. Rev. B 76, 012408 (2007).

[9] X. G. Huang, M. Matsuo, and H. Taya, Spontaneous generation of spin current from the vacuum by strong electric fields, Prog. Theor. Exp. Phys. (2019), 113B02.

[10] J.C. Collins, Renormalization (Cambridge University Press, Cambridge, England, 1986).

[11] $c / \hbar^{2}=c \times 1 / \hbar^{4} \times \hbar^{2}$, where $c$ comes from the velocity $v^{\mu}=c \alpha^{\mu}, 1 / \hbar^{4}$ comes from the momentum integration $d^{4} p /(2 \pi \hbar)^{4}$, and $\hbar^{2}$ comes from the propagator $i \hbar /(\not p-m)$.

[12] J. Schwinger, On gauge invariance and vacuum polarization, Phys. Rev. 82, 664 (1951).

[13] J. Schwinger, The theory of quantized fields. V, Phys. Rev. 93, 615 (1954); The theory of quantized fields. VI, Phys. Rev. 94, 1362 (1954).

[14] See Sec. 94 "Grand Unified Theories" of P. A. Zyla et al. (Particle Data Group), Review of particle physics, Prog. Theor. Exp. Phys. 2020, 083 C01 (2020).

[15] J. Smit, The spontaneous Hall effect in ferromagnetics I, Physica (Amsterdam) 21, 877 (1955);

[16] J. Smit, The spontaneous Hall effect in ferromagnetics II, Physica (Amsterdam) 24, 39 (1958).

[17] L. Berger, Side-jump mechanism for the Hall effect of ferromagnets, Phys. Rev. B 2, 4559 (1970);

[18] L. Berger, Application of the side-jump model to the Hall effect and Nernst effect in ferromagnets, Phys. Rev. B 5, 1862 (1972).

[19] C.S. Chu and R.X. Miao, Fermion condensation induced by the Weyl anomaly, Phys. Rev. D 102, 046011 (2020).

[20] C. S. Chu and R. X. Miao, Weyl Anomaly induced Fermi condensation and holography, J. High Energy Phys. 08 (2020) 134.

[21] L. D. Landau, L. P. Pitaevskii, and E. M. Lifshitz, Electrodynamics of Continuous Media, 2nd ed. (ButterworthHeinemann, Washington, DC, 1984).

[22] D. A. T. Somers and J. N. Munday, Rotation of a liquid crystal by the Casimir torque, Phys. Rev. A 91, 032520 (2015).

[23] D. A. T. Somers, J. L. Garrett, K. J. Palm, and J. N. Munday, Measurement of the Casimir torque, Nature (London) 564, 386 (2018).

[24] Our analysis is different from that of Ref. [22]. In Ref. [22], there is no external torque acting on the bulk of the liquid 
crystal and the equilibrium configuration is obtained by balancing the Casimir torque with a restoring elastic torque defined at the surface of the liquid crystal. As there is no torque in the bulk, the equation of motion (36) then give rises to a $\theta$ linear in $z$, which is also obtained in Ref. [22] with a different argument.

[25] I. C. Khoo, Liquid Crystals, 2nd ed. (John Wiley \& Sons, New York, 2007). 